\title{
Interplay between Electrolyte-Gated Organic Field-Effect Transistors and Surfactants: A Surface Aggregation Tool and Protecting Semiconducting Layer
}

\author{
Qiaoming Zhang," Adrián Tamayo," Francesca Leonardi,* and Marta Mas-Torrent*
}

Cite This: ACS Appl. Mater. Interfaces 2021, 13, 30902-30909

Read Online

ACCESS

Џlll Metrics \& More

回国 Article Recommendations

S1 Supporting Information

ABSTRACT: Molecular surfactants, which are based on a water-insoluble tail and a water-soluble head, are widely employed in many areas, such as surface coatings or for drug delivery, thanks to their capability to form micelles in solution or supramolecular structures at the solid/liquid interface. Electrolyte-gated organic field-effect transistors (EGOFETs) are highly sensitive to changes occurring at their electrolyte/gate electrode and electrolyte/organic semiconductor interfaces, and hence, they have been much explored in biosensing due to their inherent amplification properties. Here, we demonstrate that the EGOFETs and surfactants can provide mutual benefits to each other. EGOFETs can be a simple and complementary tool to study the aggregation behavior of cationic and anionic surfactants at low concentrations on a polarized metal surface. In this way, we have monitored the monolayer formation of cationic and anionic surfactants at the water/electrode interface with p-type and n-type devices, respectively. On the other hand, the operational stability of EGOFETs has been dramatically enhanced, thanks to the formation of a protective layer on top of the organic semiconductor by exposing it to a high concentration of a surfactant solution (above the critical micelle concentration). Stable performances were achieved for more than 10 and $2 \mathrm{~h}$ of continuous operation for p-type and n-type devices, respectively. Accordingly, this work points not only that EGOFETs can be applied to a wider range of applications beyond biosensing but also that these devices can effectively improve their long-term stability by simply treating them with a suitable surfactant.

KEYWORDS: electrolyte-gated organic field-effect transistors, surfactant, surface aggregation, protective top layer, long-term stability

\section{INTRODUCTION}

Surfactants are amphiphilic molecules that contain a hydrophilic polar head and a long hydrophobic tail. ${ }^{1-5}$ They are widely employed in many areas such as detergents and emulsifiers, in nano- and micro-particle preparation, and even in protein research and DNA extraction. ${ }^{1,6-8}$ The most intriguing property of surfactants is their capability to form supramolecular structures in a solution like micelles and selfassembled structures at the solid/liquid or solid/air interfaces like admicelles, hemimicelles, and monolayers. ${ }^{1}$ The aggregation of surfactants strongly depends on the concentration, ${ }^{2}$ electrolyte ionic strength, ${ }^{3}$ temperature, ${ }^{9}$ and surface properties. ${ }^{10} \mathrm{~A}$ range of different techniques, including vibrational sum frequency generation (SFG) spectroscopy combined with total internal reflection Raman (TIR Raman) scattering ${ }^{1}$ or with surface plasmon resonance $(\mathrm{SPR})^{2}$ and atomic force microscopy (AFM), ${ }^{3,11}$ are commonly employed to investigate the aggregation of this class of molecules on surfaces. However, these techniques are not easily available and, further, the data interpretation can be complex.

Organic electronic devices are raising considerable interest for applications requiring low cost since organic semi- conductors (OSCs) can be printed on flexible substrates over large areas. In particular, electrolyte-gated organic fieldeffect transistors (EGOFETs) are appealing for working in aqueous media. ${ }^{12-14}$ Their layout consists of exposing directly the OSC toward an electrolyte, where a gate contact is also immersed. The application of a source-gate voltage yields the formation of two electrical double layers (EDLs): one placed at the gate/electrolyte interface and the other one at the OSC/ electrolyte interface. These EDLs, which exhibit high capacitance values on the order of tens of $\mu \mathrm{F} \cdot \mathrm{cm}^{-2}$, determine the device's electrical performance. ${ }^{15}$ For this reason, EGOFETs have often been exploited as electrical transducers for biosensing by the proper modification of such interfaces with receptor groups. ${ }^{16-18}$ Additionally, although much less

Received: March 31, 2021

Accepted: June 8, 2021

Published: June 22, 2021 
(a)

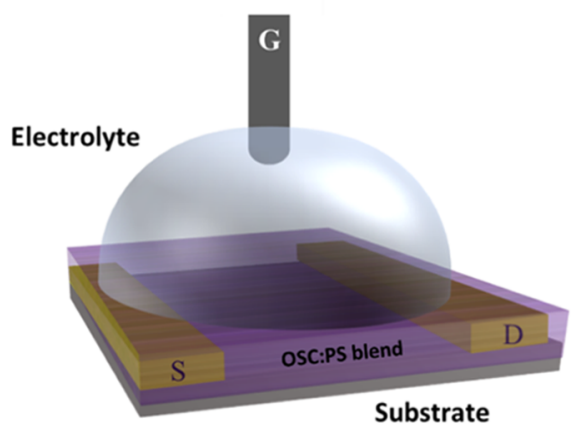

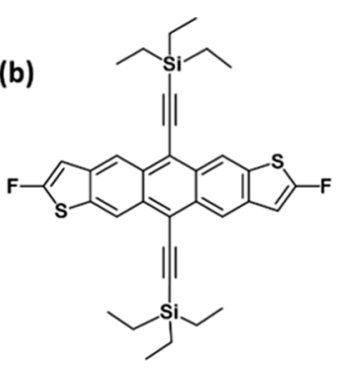

diF-TES-ADT

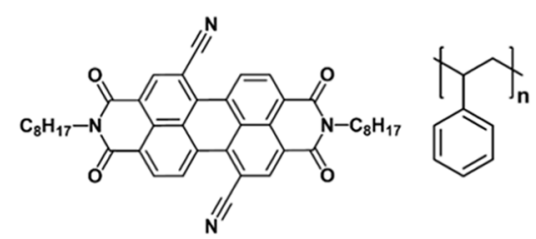

PDI8CN2

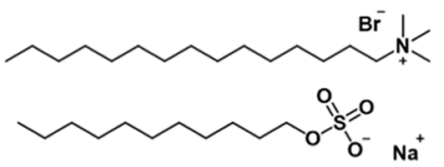

(c) Approach I

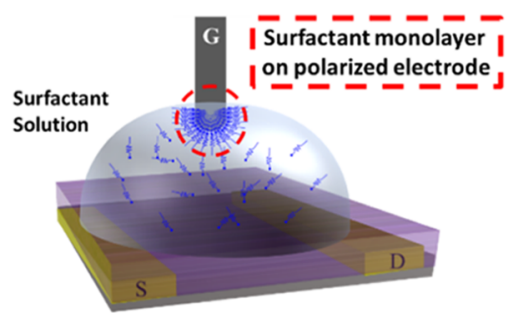

(d) Approach II

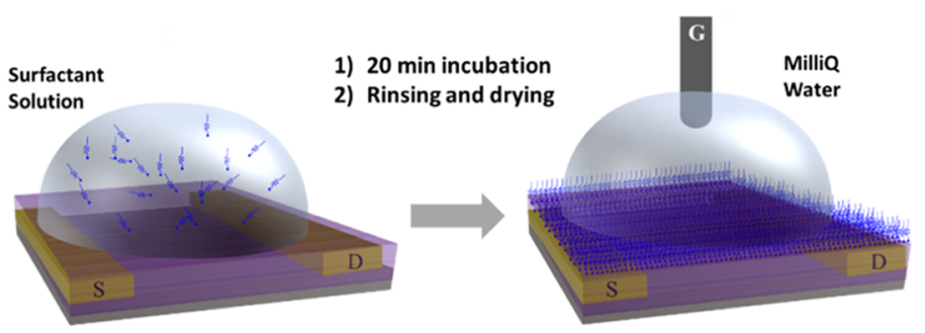

Figure 1. (a) Scheme of the EGOFET structure. (b) Molecular structures of the OSCs, PS, and surfactants used in this work. Schematic detection procedure of (c) approach I and (d) approach II.

explored, EGOFETs can also be employed as a research tool to study chemical/physical processes or to monitor cell/microorganism activities, ${ }^{19-21}$ where such events can be followed by registering an easily readable electrical signal. However, despite the high potential of these devices, some unsolved problems are hindering their technological transfer such as their longterm electrical stability in aqueous media.

In this work, we demonstrate the reciprocal benefits that surfactants and EGOFETs can offer to each other. First, we show that EGOFETs can become an appealing alternative tool to investigate the adsorption behavior of cationic and anionic surfactants on a polarized metal surface (i.e., gate electrode) by p- or n-type EGOFETs, respectively. Second, we demonstrate unprecedented long-term operation stability of EGOFETs upon exposing the OSC layer to a high concentration (above the critical micelle concentration, CMC) of a surfactant solution due to the formation of a protective top layer on the OSC. Hence, our current work further evidences the high potential of EGOFETs, proving that these devices can exhibit improved electrical performance and that they can be applied to a broader range of applications.

\section{RESULTS AND DISCUSSION}

The EGOFET architecture is shown in Figure 1a. It consists of an OSC layer bottom-contacted by two gold electrodes, namely, source (S) and drain (D). The OSC is in electric contact with a third Pt gate electrode (G) through an electrolyte. These devices operate at a very low operation voltage $(<1 \mathrm{~V})$ due to the high capacitance of electrical double layers. The application of source-gate voltage $\left(V_{\mathrm{GS}}\right)$ promotes the formation of two EDLs at the electrolyte/OSC and electrolyte/gate electrode interfaces, ${ }^{12,15}$ where the applied $V_{\mathrm{GS}}$ decreases steeply. ${ }^{22,23}$ The OSC represents the core element of this device. To obtain devices that are electrically stable in water, highly crystalline and homogeneous OSC thin films are required to avoid electrochemical doping. Previously, we showed that the deposition of blends of OSCs with an insulating polymer (i.e., polystyrene, PS) by the solution shearing technique (i.e., bar-assisted meniscus shearing, BAMS) is an effective strategy to realize high-performance EGOFET devices. ${ }^{12,15}$ This was ascribed to the high degree of thin-film crystallinity along with an extended homogeneity at the long range (i.e., from several to hundreds of micrometers). Therefore, in this work, we have followed the same approach and, as active materials, used PS-based blends of p-type OSC 2,8-difluoro-5,11-bis(triethylsilylethynyl)anthradithiophene (diF-TES-ADT) and n-type OSC N,N'-bis(n-octyl)-dicyanoperylene-3,4:9,10-bis-(dicarboximide) (PDI8CN2). The chemical structures of these materials are shown in Figure $1 \mathrm{~b}$ (see Section 4 for further details regarding the deposition of the materials and device fabrication).

Regarding surfactants, we selected cationic surfactant cetyltrimethylammonium bromide (CTAB) and anionic surfactant sodium dodecyl sulfate (SDS) (Figure 1b, bottom). Surfactants show different aggregation behaviors according to their concentration. The critical micelle concentration (CMC) refers to the minimum concentration required for micelle formation in solution, which can then assemble in a different fashion once a surface is available. At high surfactant concentrations above CMC, the morphology of surfactant aggregates on the solid surface vary from globular micelles to cylindrical micelles and, finally, to layered films. ${ }^{11}$ At the other extreme, the monolayer formation concentration (MFC), which for a given surfactant is typically several orders of magnitude below its CMC, does not allow the formation of 
micelles in solution and, at the solid-liquid interface normally promotes the formation of a molecular monolayer. ${ }^{1,2,4,6}$

2.1. Approach I: EGOFETs as a Tool to Study the Monolayer Formation on a Polarized Metal Surface. In this part of the work, we aimed at exploiting EGOFETs as an ultrasensitive tool to investigate the monolayer formation of surfactants at the water-metal interface, specifically on the EGOFET polarized $\mathrm{Pt}$ gate contact. For this purpose, to monitor the cationic CTAB surfactant on a negatively charged electrode, a p-type EGOFET was required. On the contrary, the anionic SDS was studied by employing an n-type EGOFET.

Figure 2a displays the typical p-type transfer characteristics ( $V_{\mathrm{GS}}$ window ranging from 0.3 to $-0.5 \mathrm{~V}$ ) of an EGOFET

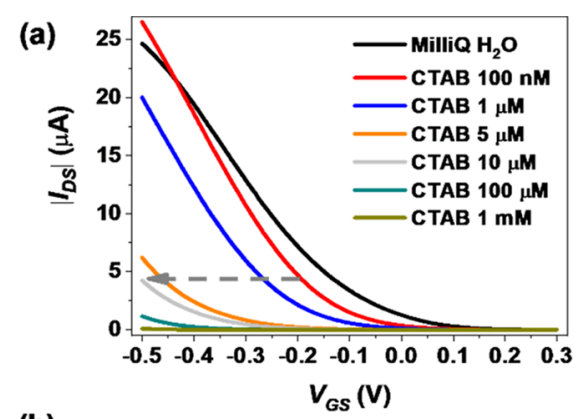

(b)

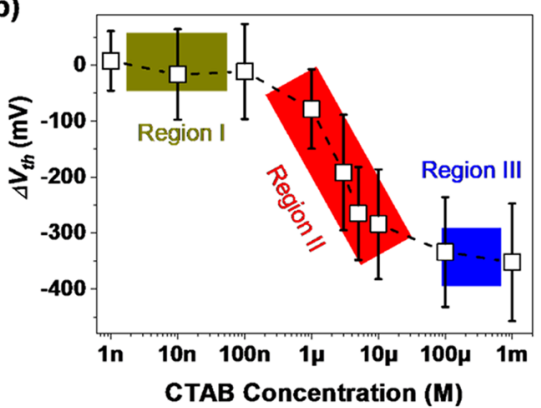

(c)

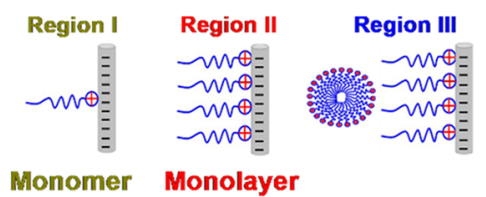

Figure 2. (a) $I-V$ transfer characteristics of p-type EGOFETs (based on diF-TES-ADT:PS blend) in the saturation regime $\left(V_{\mathrm{GS}}=-0.4 \mathrm{~V}\right)$ using CTAB solutions in Milli- $\mathrm{Q}$ water as the electrolyte. (b) Average threshold voltage shifts $\left(\Delta V_{\text {th }}\right)$ plotted against different concentrations of CTAB. These data were extracted from five devices. The EGOFET devices were exposed to $\mathrm{CTAB}$ solutions with concentrations ranging from $1 \mathrm{nM}$ to $1 \mathrm{mM}$ in ascending order. (c) Schematic picture of $\mathrm{CTA}^{+}$aggregation on the gate Pt surface.

based on diF-TES-ADT:PS using Milli-Q water as electrolyte media (black curve). A stability check consisting of recording several transfer characteristics was always performed to ensure a reproducible electrical response before the subsequent surfactant detection test (Figure S1a, Supporting Information). Afterward, water solutions with different concentrations of CTAB spanning from $1 \mathrm{nM}$ to $1 \mathrm{mM}$ were directly employed as electrolyte media in ascending order (Figure 1c, approach I). For each concentration, an additional stability check in different $\mathrm{CTAB}$ concentrations was carried out to ensure the stability and reproducibility of the data (see, for instance, the device response with $1 \mu \mathrm{M}$ CTAB in Figure S1b, Supporting Information). Clearly, transfer characteristics are significantly affected by the addition of CTAB in the media, as evidenced by the negative $V_{\text {th }}$ shift accompanied by a decrease in the source-drain current $\left(I_{\mathrm{DS}}\right)$ in the saturation regime (Figure $2 a)$. It is also worth noting that the slopes of transfer curves ( $\sqrt{I_{\mathrm{DS}}}$ vs $V_{\mathrm{GS}}$ curves) are not significantly altered in the whole experiment (Figure S1c-d, Supporting Information), indicating that product [mobility $\times$ capacitance] $\left(\mu \times c_{\mathrm{dl}}\right)$ is not much influenced (the average value is $0.23 \mu \mathrm{S} \cdot \mathrm{V}^{-1}$ with $21 \%$ variation). The same scenario is found when plotting the electrical characteristics in the linear regime $\left(V_{\mathrm{DS}}=-0.1 \mathrm{~V}\right)$ (Figure S2, Supporting Information). These observations can be rationalized by considering the aggregation behavior of the $\mathrm{CTAB}$ cations (hereafter referred to as $\mathrm{CTA}^{+}$) on the negatively polarized $\mathrm{Pt}$ gate surface. This phenomenon leads to effective screening of the gate bias, which results in a negative shift of $V_{\mathrm{th}}$, and thus, a higher $V_{\mathrm{GS}}$ is required to create a conducting channel in the OSC. The same experiment was repeated by replacing $\mathrm{CTAB}$ with $\mathrm{NaBr}$ to keep the same anion while dramatically changing the cationic counterpart. In this case, a much less pronounced negative $V_{\text {th }}$ shift was observed (see Figure S3, Supporting Information). This supports our previous assumption regarding the self-organization of the large $\mathrm{CTA}^{+}$cations on the gate electrode, which causes a stronger screening of the $V_{\mathrm{GS}}$.

To quantify the dependence of $V_{\text {th }}$ shift with respect to CTAB concentration, $V_{\text {th }}$ was extracted in linear and saturation regimes (Section 4). The $V_{\text {th }}$ shift $\left(\Delta V_{\text {th }}\right)$ is defined as $\Delta V_{\text {th }}=$

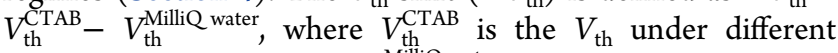

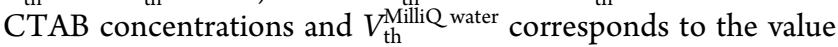
extracted when Milli-Q water is employed as electrolyte media before the addition of the surfactant. The relationship between $\Delta V_{\text {th }}$ and $\log [\mathrm{CTAB}]$ is depicted in Figure $2 \mathrm{~b}$, where three different regions can be clearly observed: a first plateau region (I) at low surfactant concentrations $(1 \mathrm{nM}<c<100 \mathrm{nM})$, a steep region (II) at medium concentrations $(100 \mathrm{nM}<c<10$ $\mu \mathrm{M})$, and a second plateau (III) at high concentrations (10 $\mu \mathrm{M}<c<1 \mathrm{mM}$ ).

The CMC of CTAB in water is known to be $1 \mathrm{mM}^{24}$ whereas its MFC is around $1-10 \mu \mathrm{M}$ in water. ${ }^{5}$ Taking this into consideration, region (I) can be attributed to the adsorption of monomers on the gate caused by electrostatic interactions between the positively charged head-group of $\mathrm{CTA}^{+}$and the negatively polarized $\mathrm{Pt}$ gate electrode, as sketched in Figure 2c. Such monomer aggregation results in negligible effects on the EGOFET response. In region (II), an abrupt $V_{\text {th }}$ shift due to effective screening of the gate bias is found, which might be indicative of the surfactant monolayer formation on the gate electrode. In region (III), above $10 \mu \mathrm{M}$, $\mathrm{CTA}^{+}$ions fully cover the surface of the Pt gate electrode, and then, an increase in surfactant concentration is no further affecting the device's electrical characteristics.

The influence of the ionic strength was also explored by phosphate-buffered solution (PBS, $1 \times, \mathrm{pH} 7.4$ ) as electrolyte media, giving a similar trend (Figure S4, Supporting Information). This result is also in agreement with the fact that, as expected, CTAB has a head-on orientation of the $\mathrm{CTA}^{+}$ions on the negatively polarized Pt surface. This can be affirmed because the Debye screening length $\left(\lambda_{\mathrm{D}}\right)$ sharply decreases with increasing ionic strength. ${ }^{6,25,26}$ At high ionic strengths, the $\mathrm{CTA}^{+}$charges would not be detected if they 

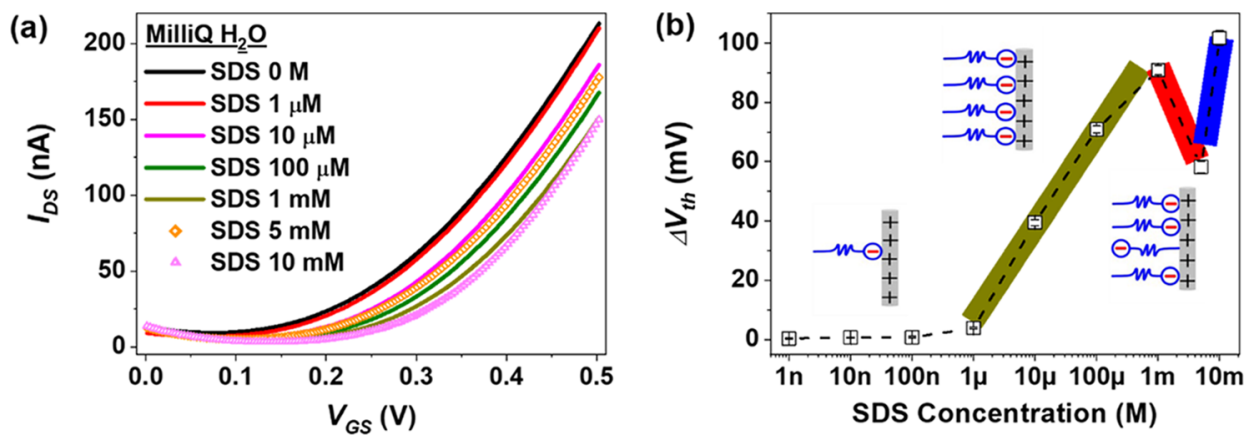

Figure 3. (a) $I-V$ characteristics in the saturation regime $\left(V_{\mathrm{DS}}=0.5 \mathrm{~V}\right)$ of an n-type EGOFET (based on PDI8CN2:PS blend) using SDS solutions of different concentrations in Milli-Q water as electrolyte media. (b) $\Delta V_{\text {th }}$ vs $\log$ [SDS] extracted from three different devices. The n-type EGOFET device was exposed to SDS surfactant solutions from $1 \mathrm{nM}$ to $10 \mathrm{mM}$ in ascending order.

were oriented far away from the Pt gate (i.e., $\lambda_{\mathrm{D}}$ is $\sim 100 \mathrm{~nm}$ in Milli-Q water, while it is $<1 \mathrm{~nm}$ in PBS $1 \times$ solution $\left.^{27}\right)$. A similar response was observed by replacing the $\mathrm{Pt}$ gate electrode with a carbon composite-based electrode (Figure S5, Supporting Information). Furthermore, cyclic voltammetry (CV) and electrochemical impedance spectroscopy (EIS) experiments further support the formation of a $\mathrm{CTA}^{+}$ monolayer on the Pt electrode (Figures S6 and S7, Supporting Information). Finally, atomic force microscopy (AFM) images on evaporated gold electrodes on Kapton after exposure to $\mathrm{CTAB}$ at a concentration of $10 \mu \mathrm{M}$ (region II) and applying a voltage sweep from 0.3 to $-0.5 \mathrm{~V}$ also confirmed the formation of a monolayer (Figure S8, Supporting Information). However, the presence of CTAB on the OSC surface was not observed under these conditions (Figure S9, Supporting Information). Noticeably, the influence of CTAB aggregation on the OSC surface was also explored by incubating the film with the surfactant previously to perform the electrical test, but not significant electrical effects were observed in the monolayer formation concentration range (Figure S10, Supporting Information).

It should be mentioned that previously an organic electrochemical transistor (OECT), whose working principle relies on doping/dedoping a conducting polymer, was reported as a suitable platform for the detection of CTAB micelles. ${ }^{28}$ This was driven by the modification of the size and type of ionic dopants occurring during $\mathrm{CTAB}$ micelle assembly. However, with the here-in fabricated EGOFETs, we are able to study the $\mathrm{CTAB}$ monolayer formation, which takes place at much lower concentrations.

To verify the versatility of this approach, the surface assembly of anionic surfactant SDS was investigated using an n-type EGOFET based on a PDI8CN2:PS blend film. ${ }^{29}$ In this device, the Pt gate electrode is positively polarized during its operation. Figure 3a displays the transfer characteristics in the saturation regime of the device exposed to different SDS concentrations. Noticeably and as the previous example, the electrical characteristics are significantly affected by the presence of the SDS surfactant due to the screening of the gate voltage by the SDS anion (hereafter referred to as $\mathrm{DS}^{-}$) assembly on the gate electrode, resulting in a $V_{\text {th }}$ shift toward higher-voltage values together with a reduction of $I_{\mathrm{DS}}$ (Figure 3a).

Analyzing in detail the $\Delta V_{\text {th }}$ shift as a function of the SDS concentration (Figure $3 \mathrm{~b}$ ), we observe that the results agree with $\mathrm{DS}^{-}$monolayer formation in the concentration range from $1 \mu \mathrm{M}$ to $1 \mathrm{mM}$. However, at higher concentrations, an oscillating trend of the $\Delta V_{\text {th }}$ is found, which seems to be in accordance with the work published by Song and co-workers, wherein SFG and SPR characterization techniques were employed. ${ }^{2}$ They found that a $\mathrm{DS}^{-}$monolayer is formed at a concentration of $0.2 \mathrm{mM}$ by the initial adsorption of $\mathrm{DS}^{-}$ molecules with their negatively charged head groups pointing toward the positively polarized surface. Nevertheless, the decrease of surface potential promotes that, at higher SDS concentrations $(3 \mathrm{mM}<c<8 \mathrm{mM})$, some SDS molecules begin adsorbing in the opposite orientation (head groups pointing toward the water phase). Such a process would explain the reduction of $V_{\mathrm{GS}}$ screening observed in our devices at SDS concentrations above $1 \mathrm{mM}$, as sketched in the inset of Figure $3 \mathrm{~b}$. The CMC of SDS is reported to be around 7-8 $\mathrm{mM}$ in deionized water; ${ }^{30}$ hence, above this concentration, micelles are formed.

All of these results have further been confirmed by EIS measurements, in which the capacitance response also exhibits an oscillating trend in the same SDS concentration range (Figure S11, Supporting Information). Noticeably, this behavior disappeared when using PBS $1 \times$ as a medium (Figure S12, Supporting Information), probably caused by the decrease of $\lambda_{\mathrm{D}}$ at high ionic strengths, ${ }^{26,31}$ which prevents the detection of the head-out orientation of $\mathrm{DS}^{-}$anions. AFM analysis of a planar gold gate electrode after being exposed to SDS at a concentration of $1 \mathrm{mM}$ and applying a transfer voltage sweep also confirmed the presence of a monolayer on the electrode (Figure S13, Supporting Information).

Thus, EGOFETs represent a promising platform to study the surface assembly of ionic surfactants at low concentrations on a polarized electrode using a simple electrical read-out.

2.2. Approach II: Surfactant Protecting Layer to Enhance the Long-Term Stability of EGOFETs. Longterm operational instability is a key factor for sensor implementation in biological and biomedical applications. ${ }^{15}$ Stability is still a major drawback in EGOFETs, and it is mainly ascribed to the migration of ions into the OSC layer. ${ }^{15}$ Therefore, finding a suitable route to protect the OSC film, without hampering the electrical performance, could be an effective way to enhance the device's stability.

It is known that when the concentrations of surfactant solutions are at least 2 times higher than their CMC, micelles aggregate on surfaces, finally forming layered films. ${ }^{11}$ Taking this into account, we proceed in using this approach for encapsulating the OSC films. Approach II consisted hence of placing a drop of the surfactant solution with a concentration above CMC on top of the OSC film for $20 \mathrm{~min}$, followed by 

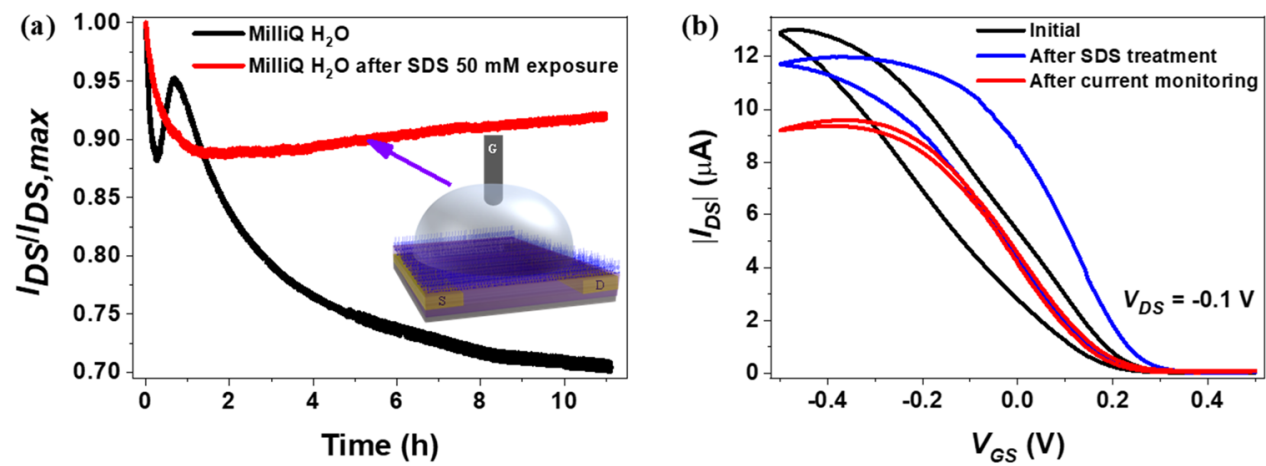

Figure 4. (a) $I-t$ plot of the normalized $I_{\mathrm{DS}}$ of a p-type EGOFET (based on diF-TES-ADT:PS blend) recorded at $V_{\mathrm{GS}}=-400 \mathrm{mV}$ and $V_{D S}=-50$ $\mathrm{mV}$ in the absence of SDS and after treating the organic semiconductor film with SDS. All data were recorded using Milli-Q water as the electrolyte. The inset is the schematic interpretation of the SDS aggregation on the diF-TES-ADT:PS blend surface. (b) $I-V$ transfer characteristics in the linear regime using Milli- $Q$ water as media recorded initially (black line), after treating the semiconductor with SDS (blue line), and after the current monitoring test of the SDS treated device (red line).
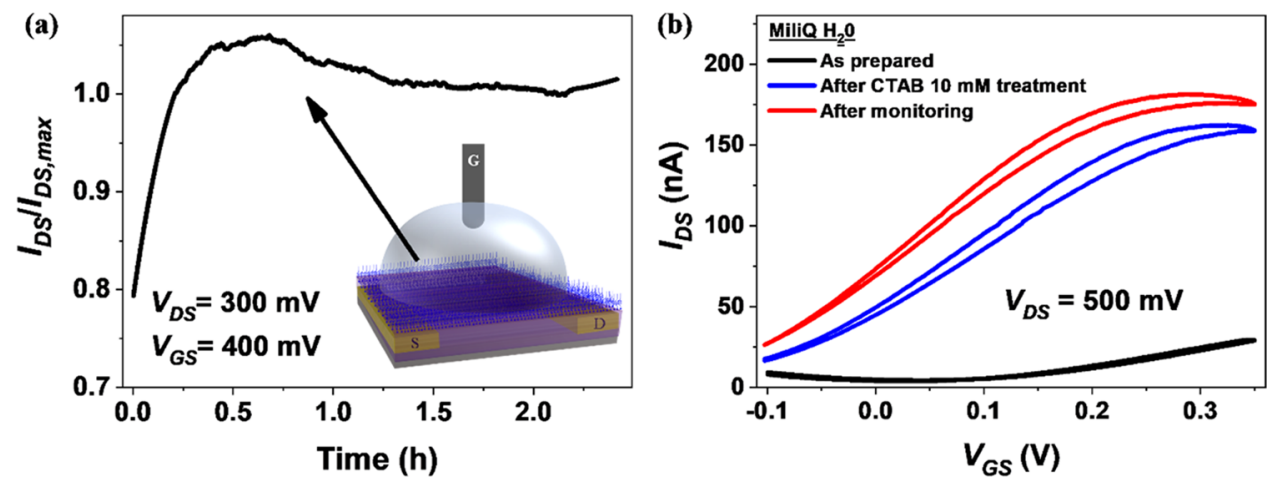

Figure 5. (a) $I-t$ plot of EGOFETs based on PDI8CN2:PS films. The normalized $I_{\mathrm{DS}}$ is recorded at fixed operation voltage $\left(V_{\mathrm{GS}}=400 \mathrm{mV}\right.$ and $V_{\mathrm{DS}}=300 \mathrm{mV}$ ) using Milli-Q water as the electrolyte and after treating the OSC film with a $10 \mathrm{mM}$ CTAB solution. The inset is the schematic view of $\mathrm{CTAB}$ aggregation on the OSC surface. (b) $I-V$ transfer characteristics recorded in the saturation regime using Milli- $\mathrm{Q}$ water as electrolyte media of the device as prepared (black line), after CTAB treatment (blue line), and after the current monitoring test (red line).

the removal of the solution and then drying with a $\mathrm{N}_{2}$ flow. Then, the EGOFET was electrically tested in Milli-Q water (Figure 1d).

The first test was performed using the p-type EGOFET based on diF-TES-ADT:PS and the anionic SDS surfactant. To optimize the SDS concentration, a series of different concentrations of SDS solutions in Milli- $Q$ water were employed. Current monitoring tests under fixed $V_{\mathrm{GS}}=-400$ $\mathrm{mV}$ and $V_{\mathrm{DS}}=-50 \mathrm{mV}$ were performed to test their protective efficacy. As displayed in Figure S14 (Supporting Information), the optimized SDS concentration was $50 \mathrm{mM}$. To highlight the long-term stability induced by the SDS layer, the normalized and original current monitoring data obtained from devices with and without the SDS treatment are presented in Figures $4 \mathrm{a}$ and S15 (Supporting Information), respectively. In the absence of SDS (black curve), the $I_{\mathrm{DS}}$ exhibits a fast decrease in the first half-hour followed by a current increase in the following $30 \mathrm{~min}$ and then a slow but continuous decrease for the remaining test time (more than $30 \%$ of the current is dropped after $10 \mathrm{~h}$ ). In contrast, the EGOFETs treated with SDS show an initial 10\% current decrease but, afterward, show a stable current value for more than $10 \mathrm{~h}$ (red curve). This enhanced and unprecedented operation stability is mainly ascribed to the protective action provided by the SDS layer formed on top of the OSC film, as sketched in the inset of Figure 4a. Our observations are further confirmed by the transfer characteristics recorded prior to and after $I / t$ monitoring. As shown in Figure $4 \mathrm{~b}$, in the transfer characteristics registered after the SDS treatment (blue line), only a small positive $V_{\text {th }}$ shift accompanied with $I_{\mathrm{DS}}$ increase is found with respect to the initial device (black line). These results are attributed to the $\mathrm{DS}^{-}$anions that aggregate on the OSC surface, inducing more holes in the conduction channel. After the current monitoring test (red line), the device shows a stable electrical performance with a slight $I_{\mathrm{DS}}$ decrease and a significantly reduced hysteresis. It should be noticed that only when the device is operated at a high source-drain bias $\left(V_{\mathrm{DS}}=\right.$ $-400 \mathrm{mV}$ ), the enhanced device stability is no more realized, which could be attributed to the high longitudinal field present along the OSC channel (Figure S16, Supporting Information).

AFM was employed to study the morphology of the OSC thin-film surface prior to and after exposure to the SDS surfactant solution. As shown in Figure S17 (Supporting Information), the AFM images reveal morphological features at the nanoscale level on top of the diF-TES-ADT:PS thin film, which can be attributed to the SDS assemblies.

Similar results were obtained using films of only diF-TES$\mathrm{ADT}$ (i.e., without the binding PS polymer) or also using another anionic surfactant such as sodium octyl sulfate (SOS) (Figures S18 and S19, Supporting Information). Finally, a comparison experiment was carried out by substituting SDS for the cationic surfactant $\mathrm{CTAB}$ following the same experimental protocol (Figure S17, Supporting Information). As displayed in Figure S20 (Supporting Information), no device stability 
improvement was noticed in this case. This indicates that with the cationic surfactant the protective layer on the OSC is not stably formed under the operational device conditions of a $\mathrm{p}$ type EGOFET, which can be ascribed to the polarization of the device (the OSC is positively charged under operation).

To further verify this and validate our methodology, PDI8CN2:PS films were exposed to CTAB (10 mM solution) and implemented as n-type EGOFET. As in the previous case, AFM characterization shows the formation of a passivating layer on top of the organic semiconductor film after exposing it to CTAB (Figure S21, Supporting Information). It is important to highlight that n-type EGOFET devices suffer much fast deterioration due to moisture and water. ${ }^{32}$ As displayed in Figure 5a, the same stability enhancement observed previously with the p-type EGOFET was encountered again here. Indeed, a sharp $I_{\mathrm{DS}}$ increase in the first halfhour followed by a constant $I_{\mathrm{DS}}$ in the following $2 \mathrm{~h}$ was found. In stark contrast, the n-type EGOFET with no protection shows fast and important deterioration with about $90 \%$ decrease of the initial current after $3 \mathrm{~h}$ (Figure S22, Supporting Information). This behavior is further confirmed by comparing the transfer characteristics prior to and after the current monitoring test (Figures $5 \mathrm{~b}$ and S22, Supporting Information). After protecting the organic semiconductor with CTAB, an important shift of the transfer characteristics takes place. Remarkably, after $3 \mathrm{~h}$ of continuous operation during the monitoring test, the transfer characteristics registered prove that the device is still working properly.

\section{CONCLUSIONS}

In conclusion, we have fabricated p-type and n-type EGOFETs based on printed OSC:PS blends and studied the effect of the surface aggregation of surfactants on their interfaces, which is highly influenced by the device polarization. First, the EGOFETs have been demonstrated to be a simple, complimentary, and highly sensitive tool to investigate the adsorption behavior of cationic (i.e., CTAB) and anionic (i.e., SDS) surfactants on the gate surface of EGOFETs. Employing a p-type EGOFET, the formation of a monolayer of the $\mathrm{CTA}^{+}$ cations on the negatively polarized $\mathrm{Pt}$ gate electrode could be monitored. This was evidenced by a sharp $V_{\text {th }}$ variation in the concentration range $(1 \mu \mathrm{M}<c<10 \mu \mathrm{M})$. Additionally, with an n-type EGOFET, the surface assembly of SDS was explored. The results are in agreement with a head-on monolayer formation up to a concentration of $1 \mathrm{mM}$. Afterward, the oscillating trend of $V_{\text {th }}$ found indicates that some SDS molecules upturn adsorbing on the surface in the opposite orientation.

In the second part of the work, surfactants were exploited to spontaneously form a protective layer for the OSC thin films to prolong EGOFETs' long-term stability. In particular, forming a layer of the anionic SDS surfactant on top of a p-type OSC unprecedentedly enhanced the device's stability for more than $10 \mathrm{~h}$ in continuous operation. Similarly, the cationic CTAB surfactant improved the water stability of an n-type OSC for more than $2 \mathrm{~h}$.

Our results point not only that EGOFETs can be applied to a wider range of applications beyond biosensing but also that these devices can exhibit long-term stability when operating in a water environment by simply treating them with a suitable surfactant. Hence, low-cost EGOFET devices might become a simple and versatile tool for analysis in laboratories covering different fields.

\section{EXPERIMENTAL SECTION}

4.1. Materials. The organic semiconductor materials used in this work, 2,8-difluoro-5,11-bias(triethylsilylethynyl)anthradithiophene (diF-TES-ADT) and $N, N^{\prime}$-bis(n-octyl)-dicyanoperylene-3,4:9,10-bis(dicarboximide) (PDI8CN2), were purchased from Lumtec and Polyera Inc. (N1200), respectively. Polystyrene (PS, $M_{\mathrm{w}}=10000$ and $\left.280000 \mathrm{~g} \cdot \mathrm{mol}^{-1}\right)$, chlorobenzene (CB), and 2,3,4,5,6-pentafluorothiophenol (PFBT) were provided by Sigma-Aldrich. The cetyltrimethylammonium bromide (CTAB) and sodium dodecyl sulfate (SDS) were also purchased from Sigma-Aldrich. All of the commercial materials were used as received without any further purification. Platinum $(\mathrm{Pt})$ wire $(\Phi=0.5 \mathrm{~mm})$ and silver $(\mathrm{Ag})$ wire $(\Phi=0.5 \mathrm{~mm})$ were obtained from Sigma-Aldrich. Multiwalled carbon nanotubes ( $>95 \%$ of carbon purity, $10-30 \mathrm{~nm}$ of outer diameter, and about $5-15 \mu \mathrm{m}$ of length) were purchased from SES Research (Houston, TX). Pt wire was cleaned in hot sulfuric acid (0.1 M) for $30 \mathrm{~min}$ and rinsed with Milli- $Q$ water prior to each electrical and electrochemical measurement. Milli- $Q$ water $(18.2 \mathrm{M} \Omega \cdot \mathrm{cm}$ resistivity at $25{ }^{\circ} \mathrm{C}$ ) was obtained from a Milli-Q Integral Water Purification System.

4.2. EGOFET Device Fabrication. The diF-TES-ADT:PS ${ }_{10 \mathrm{~K}}$ (4:1) and PDI8CN2:PS ${ }_{280 \mathrm{~K}}(1: 2)$ blend solutions used in this work were prepared according to our previously published protocol. ${ }^{15,29}$ The $S / D$ electrodes $(\mathrm{Cr} / \mathrm{Au}, 5 / 40 \mathrm{~nm})$, with a geometrical ratio $W / L$ $=690$ (channel width $W=20700 \mu \mathrm{m}$ and channel length $L=30$ $\mu \mathrm{m})$, were deposited on Kapton polyimide film (75 $\mu \mathrm{m}$ thick, DuPont) by a thermal evaporation system (Evaporation System Auto 306 from Boc Edwards) at a pressure of $2 \times 10^{-4} \mathrm{~Pa}$. Prior to the deposition of the OSC:PS blend solution, the $S / D$ substrates were cleaned in an ultrasonic bath with acetone and isopropanol for $15 \mathrm{~min}$ each, followed by an ozone treatment for $25 \mathrm{~min}$. After that, the cleaned $S / D$ electrodes were functionalized by PFBT through immersion of the substrate into PFBT/isopropanol $(2 \mu \mathrm{L}: 1 \mathrm{~mL})$ solution for $15 \mathrm{~min}$ and then rinsed with isopropanol. Finally, all of the OSC:PS blend films were coated by the bar-assisted meniscus shearing (BAMS) technique at a fixed speed of $1 \mathrm{~cm} \cdot \mathrm{s}^{-1}$ and a plate temperature of $105^{\circ} \mathrm{C}$ in ambient conditions as previously reported..$^{15}$

4.3. Surfactant Solution Preparation. Surfactant solutions with different concentrations were prepared in Milli- $Q$ water and phosphate-buffered saline (PBS $1 \times$, pH 7.4 with $137 \mathrm{mM} \mathrm{NaCl}, 2.7$ $\left.\mathrm{mM} \mathrm{KCl}, 10 \mathrm{mM} \mathrm{Na} 2 \mathrm{HPO}_{4}, 2 \mathrm{mM} \mathrm{KH_{2 }} \mathrm{PO}_{4}\right)$. All of the original surfactant solutions were prepared at a concentration of $100 \mathrm{mM}$ by dissolving the corresponding surfactant salts and then stirring at $40{ }^{\circ} \mathrm{C}$ for at least $1 \mathrm{~h}$ to completely dissolve the powder. Afterward, the solutions were diluted as required with Milli-Q water or PBS solution. All of the surfactant solutions were freshly prepared before each experiment.

4.4. Electrical Measurements. Electrical measurements, including transfer, output characteristics, and long-term stability tests, were recorded in ambient conditions using an Agilent B1500A semiconductor device analyzer connected to the samples with a Karl SÜSS probe station. For the surfactant aggregation behavior study (approach I), a poly(dimethylsiloxane) (PDMS) pool was placed on top of the interdigitated area of the EGOFETs to confine $30 \mu \mathrm{L}$ of target aqueous solution as electrolyte media and a gate electrode $(\mathrm{Pt}$ wire, carbon paste electrode), as depicted in Figure 1c. The surfactants, dissolved in Milli- $Q$ water or PBS $1 \times$ solution, were employed directly as electrolyte media during the EGOFET tests in a concentration spanning from nanomolar to millimolar. Carbon paste electrode was fabricated according to a previously reported protocol. $^{33,34}$ The scan rate of transfer characteristics was set to $\sim 70 \mathrm{mV} \cdot \mathrm{s}^{-1}$ unless otherwise specified.

To investigate the in situ long-term EGOFET monitoring after the formation of a surfactant protecting layer on top of the OSC (approach II), a drop of surfactant solution $(\sim 50 \mu \mathrm{L})$ with target concentrations $(1,5,10,50$, and $100 \mathrm{mM})$ was placed on top of the active area and kept for 20 min under ambient conditions to allow the self-assembly of a compact surfactant layer on top of the semiconductor (Figure 1d, left). Then, the solution was removed 
by gentle rinsing and dried with $\mathrm{N}_{2}$. A large PDSM pool $(\sim 500 \mu \mathrm{L})$ was fixed on top of the interdigitated area followed by the injection of Milli-Q water into the pool as electrolyte media (Figure 1d, right). During the long-term stability test, a parafilm was sealed on top of the pool to minimize solvent evaporation during the test. $I_{\mathrm{DS}}$ was continuously recorded with an interval time of $1 \mathrm{~s}$ at $V_{\mathrm{GS}}=-400 \mathrm{mV}$ and $V_{\mathrm{DS}}=-50$ or $-100 \mathrm{mV}$ for p-type devices, whereas for n-type devices, we applied $V_{\mathrm{GS}}=500 \mathrm{mV}$ and $V_{\mathrm{DS}}=100 \mathrm{mV}$.

To quantitatively describe the properties of EGOFETs, the classical MOSFET model has been employed to extract the figure of merits, including the threshold voltage $\left(V_{\text {th }}\right)$, total electrical double layers capacitance $\left(C_{\mathrm{dl}}\right)$, and charge carrier mobility $(\mu)$. According to the MOSFET model, the $I_{\mathrm{DS}}$ values in linear and saturation regimes are approximately determined by the following equations

$$
\begin{aligned}
& I_{\mathrm{DS}}=\frac{W}{L} c_{\mathrm{d} 1} \mu\left(V_{\mathrm{GS}}-V_{\mathrm{th}}\right) V_{\mathrm{DS}}(\text { linear }) \\
& I_{\mathrm{DS}}=\frac{W}{2 L} c_{\mathrm{d} l} \mu\left(V_{\mathrm{GS}}-V_{\mathrm{th}}\right)^{2} \text { (saturation) }
\end{aligned}
$$

where $W$ is the channel width and $L$ is the channel length.

4.5. Morphological Characterization. Atomic force microscopy (AFM) images were obtained working with a 5100 SPM system from Agilent Technologies in tapping mode. For approach I, gold gate electrodes were evaporated on Kapton and analyzed by AFM after being exposed to ML and CMC concentration of CTAB (10 $\mu \mathrm{M}$ and $10 \mathrm{mM}$, respectively) and SDS (1 and $50 \mathrm{mM}$, respectively) while a voltage sweep was applied (from 0.3 to $-0.5 \mathrm{~V}$ for $\mathrm{CTAB}$ and from -0.3 to $0.5 \mathrm{~V}$ for SDS) to them using a Novocontrol Alpha-AN impedance analyzer equipped with POT/GAL 30V/2A. To check the formation of the surfactant protective layer on the OSC thin film, the freshly prepared OSC thin films were first investigated. Then, the samples were dipped into a surfactant solution at a concentration above its CMC for $20 \mathrm{~min}$ and then slightly rinsed and dried with $\mathrm{N}_{2}$ gas. All images were recorded in ambient conditions and analyzed using Gwyddion 2.47 software.

4.6. Electrochemical Impedance Spectroscopy (EIS). Cyclic voltammetry (CV) and electrochemical impedance spectroscopy (EIS), including capacitance and phase angle spectra, were carried out using a Novocontrol Alpha-AN impedance analyzer equipped with a POT/GAL $30 \mathrm{~V} / 2 \mathrm{~A}$ electrochemical interface in a frequency range between $10^{5}$ and $10^{-1} \mathrm{~Hz}$. CV measurements were carried out in a conventional three-electrode electrochemical cell using a Pt wire as the working electrode $(\Phi=0.5 \mathrm{~mm})$, a second Pt wire as the counter electrode $(\Phi=0.5 \mathrm{~mm})$, and an $\mathrm{Ag} / \mathrm{AgCl}$ quasi-reference electrode $(\Phi=0.5 \mathrm{~mm})$. Electrochemical responses were recorded in $0.1 \mathrm{M} \mathrm{KCl}$ aqueous solution containing $0.1 \mathrm{mM}\left[\mathrm{Fe}(\mathrm{CN})_{6}\right]^{3-/ 4-}$ as the redox marker. During the $\mathrm{CV}$ test, a $100 \mathrm{mM}$ CTAB solution in Milli- $Q$ water was added to the electrochemical cell to achieve the desired concentration of $C T A B$ in the working solution. The scan rate of the $\mathrm{CV}$ measurements was set to $10 \mathrm{mV} \cdot \mathrm{s}^{-1}$. For the impedance measurements, a square Au electrode $\left(S=1 \mathrm{~mm}^{2}\right)$ was employed as the counter electrode, a Pt wire $(\Phi=0.5 \mathrm{~mm})$ served as the working electrode, and $\mathrm{Ag} / \mathrm{AgCl}$ wire $(\Phi=0.5 \mathrm{~mm})$ was used as the quasireference electrode. Surfactant solutions of different concentrations (in ascending order) were used as media confined with a PDMS pool on the counter electrode. Prior to each EIS test, the CV measurement was implemented to guarantee the migration and adsorption of $\mathrm{CTA}^{+}$ or $\mathrm{DS}^{-}$on the Pt surface, which was obtained by defining the DC voltage in the same window $(0.3$ to $-0.5 \mathrm{~V}$, a step of $-0.1 \mathrm{~V}$ in the CTAB case, while 0 to $0.5 \mathrm{~V}$, a step of $0.1 \mathrm{~V}$ in the SDS case) used for the EGOFET transfer characteristics. Then, the surfactant solutions were replaced by Milli- $Q$ water. During EIS tests, DC and AC signals were fixed to 0 and $20 \mathrm{mV}$, respectively.

\section{ASSOCIATED CONTENT}

\section{(s) Supporting Information}

The Supporting Information is available free of charge at https://pubs.acs.org/doi/10.1021/acsami.1c05938.
Additional electrical transfer characteristics of EGOFETs, CV and EIS characterizations, AFM images, determination of the optimized surfactant concentration for the formation of a protective layer on top of OSC in p-type EGOFETs, and additional $I-t$ stability plots (PDF)

\section{AUTHOR INFORMATION}

\section{Corresponding Authors}

Francesca Leonardi - Institut de Ciencia de Materials de Barcelona, ICMAB-CSIC, 08193 Barcelona, Spain; Present Address: OnePlanet Research Center, Wageningen

Campus, Plus Ultra II, Bronland 10, $6708 \mathrm{WH}$

Wageningen, The Netherlands.;

Email: Francesca.Leonardi@imec.nl

Marta Mas-Torrent - Institut de Ciencia de Materials de Barcelona, ICMAB-CSIC, 08193 Barcelona, Spain; 다이.org/0000-0002-1586-005X; Email: mmas@ icmab.es

\section{Authors}

Qiaoming Zhang - School of Physical Science and Technology, Southwest University, 400715 Chongqing, P. R. China; Institut de Ciencia de Materials de Barcelona, ICMAB-CSIC, 08193 Barcelona, Spain

Adrián Tamayo - Institut de Ciencia de Materials de Barcelona, ICMAB-CSIC, 08193 Barcelona, Spain

Complete contact information is available at: https://pubs.acs.org/10.1021/acsami.1c05938

\section{Author Contributions}

"Q.Z. and A.T. equally contributed to this work.

\section{Notes}

The authors declare no competing financial interest.

\section{ACKNOWLEDGMENTS}

This work was funded by DGI (Spain) through project GENESIS PID2019-111682RB-I00, the Generalitat de Catalunya (2017-SGR-918), and the Spanish Ministry of Economy and Competitiveness through the "Severo Ochoa" Programme for Centers of Excellence in R\&D (FUNFUTURE CEX2019000917-S). A.T. acknowledges the FPU fellowship and is enrolled in the $\mathrm{UAB}$ Materials Science $\mathrm{PhD}$ program. F.L. gratefully acknowledges the "Juan de la Cierva" programme.

\section{REFERENCES}

(1) Tyrode, E.; Rutland, M. W.; Bain, C. D. Adsorption of CTAB on Hydrophilic Silica Studied by Linear and Nonlinear Optical Spectroscopy. J. Am. Chem. Soc. 2008, 130, 17434-17445.

(2) Song, S. H.; Koelsch, P.; Weidner, T.; Wagner, M. S.; Castner, D. G. Sodium Dodecyl Sulfate Adsorption onto Positively Charged Surfaces: Monolayer Formation with Opposing Headgroup Orientations. Langmuir 2013, 29, 12710-12719.

(3) Sakai, H.; Nakamura, H.; Kozawa, K.; Abe, M. Atomic Force Microscopy Observation of the Nanostructure of Tetradecyltrimethylammonium Bromide Films Adsorbed at the Mica/Solution Interface. Langmuir 2001, 17, 1817-1820.

(4) Jiménez-Ángeles, F.; Khoshnood, A.; Firoozabadi, A. Molecular Dynamics Simulation of the Adsorption and Aggregation of Ionic Surfactants at Liquid-Solid Interfaces. J. Phys. Chem. C 2017, 121, $25908-25920$.

(5) Hu, C.; Dang, X.; Hu, S. Studies on Adsorption of Cetyltrimethylammonium Bromide at Carbon Paste Electrode and the Enhancement Effect in Thyroxine Reduction by Voltammetry and 
Electrochemical Impedance Spectroscopy. J. Electroanal. Chem. 2004, 572, 161-171.

(6) Atkin, R.; Craig, V. S. J.; Wanless, E. J.; Biggs, S. Mechanism of Cationic Surfactant Adsorption at the Solid-Aqueous Interface. Adv. Colloid Interface Sci. 2003, 103, 219-304.

(7) Tsagkaropoulou, G.; Allen, F. J.; Clarke, S. M.; Camp, P. J. SelfAssembly and Adsorption of Cetyltrimethylammonium Bromide and Didodecyldimethylammonium Bromide Surfactants at the MicaWater Interface. Soft Matter 2019, 15, 8402-8411.

(8) Hideshima, S.; Saito, M.; Fujita, K.; Harada, Y.; Tsuna, M.; Sekiguchi, S.; Kuroiwa, S.; Nakanishi, T.; Osaka, T. Label-Free Detection of Allergens in Food via Surfactant-Induced Signal Amplification Using a Field Effect Transistor-Based Biosensor. Sens. Actuators, B 2018, 254, 1011-1016.

(9) Kawai, T.; Kamio, H.; Kondo, T.; Kon-No, K. Effects of Concentration and Temperature on SDS Monolayers at the Air Solution Interface Studied by Infrared External Reflection Spectroscopy. J. Phys. Chem. B 2005, 109, 4497-4500.

(10) Islam, M. N.; Sharker, K. K.; Sarker, K. C. Salt-Induced Modulation of the Krafft Temperature and Critical Micelle Concentration of Benzyldimethylhexadecylammonium Chloride. J. Surfactants Deterg. 2015, 18, 651-659.

(11) Zhao, F.; Du, Y.; Yang, P.; Li, X.; Tang, J. Adsorption Behavior of Hexadecyltrimethylammonium Bromide (CTAB) to Mica Substrates as Observed by Atomic Force Microscopy. Sci. China, Ser. B: Chem. 2005, 48, 101-106.

(12) Leonardi, F.; Casalini, S.; Zhang, Q.; Galindo, S.; Gutiérrez, D.; Mas-Torrent, M. Electrolyte-Gated Organic Field-Effect Transistor Based on a Solution Sheared Organic Semiconductor Blend. Adv. Mater. 2016, 28, 10311-10316.

(13) Liao, C.; Yan, F. Organic Semiconductors in Organic Thin-Film Transistor-Based Chemical and Biological Sensors. Polym. Rev. 2013, 53, 352-406.

(14) Torsi, L.; Magliulo, M.; Manoli, K.; Palazzo, G. Organic FieldEffect Transistor Sensors: A Tutorial Review. Chem. Soc. Rev. 2013, $42,8612-8628$.

(15) Zhang, Q.; Leonardi, F.; Casalini, S.; Temiño, I.; Mas-Torrent, M. High Performing Solution-Coated Electrolyte-Gated Organic Field-Effect Transistors for Aqueous Media Operation. Sci. Rep. 2016, 6, No. 39623.

(16) Manoli, K.; Magliulo, M.; Mulla, M. Y.; Singh, M.; Sabbatini, L.; Palazzo, G.; Torsi, L. Printable Bioelectronics to Investigate Functional Biological Interfaces. Angew. Chem., Int. Ed. 2015, 54, 12562-12576.

(17) Casalini, S.; Dumitru, A. C.; Leonardi, F.; Bortolotti, C. A.; Herruzo, E. T.; Campana, A.; De Oliveira, R. F.; Cramer, T.; Garcia, R.; Biscarini, F. Multiscale Sensing of Antibody-Antigen Interactions by Organic Transistors and Single-Molecule Force Spectroscopy. ACS Nano 2015, 9, 5051-5062.

(18) Ricci, S.; Casalini, S.; Parkula, V.; Selvaraj, M.; Deniz, G.; Greco, P.; Biscarini, F.; Mas-Torrent, M. Label-Free Immunodetection of $\alpha$-Synuclein by Using a Microfluidics Coplanar ElectrolyteGated Organic Field-Effect Transistor. Biosens. Bioelectron. 2020, 167, No. 112433.

(19) Le Gall, J.; Mouillard, F.; Le, T. N.; Vu, T. T.; Mattana, G.; Brayner, R.; Zrig, S.; Noël, V.; Piro, B. Monitoring Photosynthetic Microorganism Activity with an Electrolyte-Gated Organic Field Effect Transistor. Biosens. Bioelectron. 2020, 157, No. 112166.

(20) Fillaud, L.; Petenzi, T.; Pallu, J.; Piro, B.; Mattana, G.; Noel, V. Switchable Hydrogel-Gated Organic Field-Effect Transistors. Langmuir 2018, 34, 3686-3693.

(21) Kyndiah, A.; Leonardi, F.; Tarantino, C.; Cramer, T.; MillanSolsona, R.; Garreta, E.; Montserrat, N.; Mas-Torrent, M.; Gomila, G. Bioelectronic Recordings of Cardiomyocytes with Accumulation Mode Electrolyte Gated Organic Field Effect Transistors. Biosens. Bioelectron. 2020, 150, No. 111844.

(22) Cramer, T.; Campana, A.; Leonardi, F.; Casalini, S.; Kyndiah, A.; Murgia, M.; Biscarini, F. Water-Gated Organic Field Effect
Transistors-Opportunities for Biochemical Sensing and Extracellular Signal Transduction. J. Mater. Chem. B 2013, 1, 3728-3741.

(23) Tibaldi, A.; Fillaud, L.; Anquetin, G.; Woytasik, M.; Zrig, S.; Piro, B.; Mattana, G.; Noël, V. Electrolyte-Gated Organic Field-Effect Transistors (EGOFETs) as Complementary Tools to Electrochemistry for the Study of Surface Processes. Electrochem. Commun. 2019, 98, 43-46.

(24) Häckl, K.; Li, H.; Aldous, I. M.; Tsui, T.; Kunz, W.; Abbott, A. P.; Warr, G. G.; Atkin, R. Potential Dependence of Surfactant Adsorption at the Graphite Electrode/Deep Eutectic Solvent Interface. J. Phys. Chem. Lett. 2019, 10, 5331-5337.

(25) Gebbie, M. A.; Dobbs, H. A.; Valtiner, M.; Israelachvili, J. N. Long-Range Electrostatic Screening in Ionic Liquids. Proc. Natl. Acad. Sci. U.S.A. 2015, 112, 7432-7437.

(26) Adibnia, V.; Shrestha, B. R.; Mirbagheri, M.; Murschel, F.; De Crescenzo, G.; Banquy, X. Electrostatic Screening Length in "Soft" Electrolyte Solutions. ACS Macro Lett. 2019, 8, 1017-1021.

(27) Kergoat, L.; Piro, B.; Berggren, M.; Pham, M. C.; Yassar, A.; Horowitz, G. DNA Detection with a Water-Gated Organic FieldEffect Transistor. Org. Electron. 2012, 13, 1-6.

(28) Tarabella, G.; Nanda, G.; Villani, M.; Coppedè, N.; Mosca, R.; Malliaras, G. G.; Santato, C.; Iannotta, S.; Cicoira, F. Organic Electrochemical Transistors Monitoring Micelle Formation. Chem. Sci. 2012, 3, 3432-3435.

(29) Campos, A.; Riera-Galindo, S.; Puigdollers, J.; Mas-Torrent, M. Reduction of Charge Traps and Stability Enhancement in SolutionProcessed Organic Field-Effect Transistors Based on a Blended nType Semiconductor. ACS Appl. Mater. Interfaces 2018, 10, 1595215961.

(30) Hu, K.; Bard, A. J. Characterization of Adsorption of Sodium Dodecyl Sulfate on Charge-Regulated Substrates by Atomic Force Microscopy Force Measurements. Langmuir 1997, 13, 5418-5425.

(31) Brown, M. A.; Goel, A.; Abbas, Z. Effect of Electrolyte Concentration on the Stern Layer Thickness at a Charged Interface. Angew. Chem., Int. Ed. 2016, 55, 3790-3794.

(32) Porrazzo, R.; Luzio, A.; Bellani, S.; Bonacchini, G. E.; Noh, Y. Y.; Kim, Y. H.; Lanzani, G.; Antognazza, M. R.; Caironi, M. WaterGated n-Type Organic Field-Effect Transistors for Complementary Integrated Circuits Operating in an Aqueous Environment. ACS Omega 2017, 2, 1-10.

(33) Muñoz, J.; Leonardi, F.; Özmen, T.; Riba-Moliner, M.; González-Campo, A.; Baeza, M.; Mas-Torrent, M. Carbon-Paste Nanocomposites as Unconventional Gate Electrodes for ElectrolyteGated Organic Field-Effect Transistors: Electrical Modulation and Bio-Sensing. J. Mater. Chem. C 2019, 7, 14993-14998.

(34) Tamayo, A.; Muñoz, J.; Mas-Torrent, M. Electronic Performance of Polymer Carbon-Paste Nanoallotropes from $0 \mathrm{D}$ to $3 \mathrm{D}$ as Novel Gate Electrodes in Water-Gated Organic Field-Effect Transistors. Adv. Electron. Mater. 2020, 6, No. 2000431. 